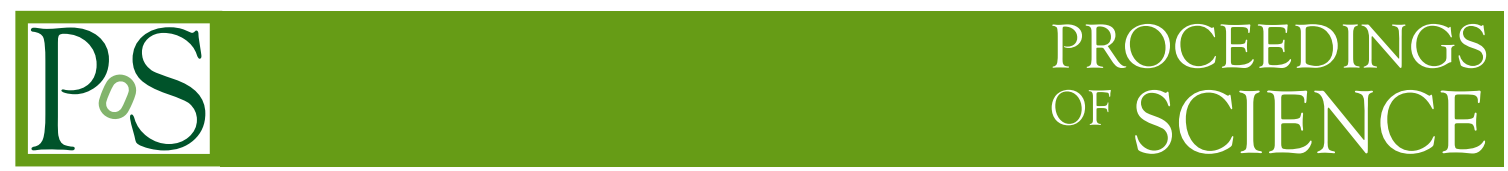

\title{
Vector meson production at large $|t|$ at HERA
}

\section{Marta Ruspa*}

University of Eastern Piedmont, Novara, and INFN-Torino, Italy

E-mail: ruspa@to.infn.it

International Europhysics Conference on High Energy Physics

July 21st - 27th 2005

Lisboa, Portugal

\footnotetext{
*Speaker.

${ }^{\dagger}$ On behalf of the H1 and ZEUS Collaborations.
} 


\section{Introduction}

The dynamics of diffractive interactions at HERA can be studied through exclusive vector meson production, $e+p \longrightarrow e+V+Y$, where $V=\rho^{0}, \omega, \phi, J / \psi, \ldots$, and $Y$ is either an elastically scattered proton or a low-mass dissociative system. In the presence of a hard scale like large values of the virtuality of the exchanged photon, $Q^{2}$, of the momentum transfer at the proton vertex, $|t|$, or of the vector meson mass, perturbative QCD (pQCD) is expected to apply. Diffractive vector meson production can then be viewed in the proton rest frame as a sequence of three processes well separated in time: the photon fluctuates into a $q \bar{q}$ pair, which then interacts with the proton via colour singlet exchange and finally recombines into a bound vector meson. The colour singlet exchange is realised in lowest order QCD by the exchange of a pair of gluons with opposite colour [1], while in the leading logarithm approximation it is described by the effective exchange of a gluonic ladder, which at sufficiently large values of $\log \left(Q^{2} / W\right)$ or $\log (-t / W)$, where $W$ is the centre-of-mass energy of the photon-proton system, is expected to include contributions from

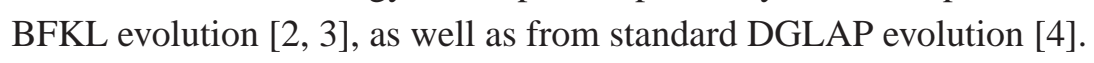

In vector meson production at large $|t|$, proton dissociative processes dominate and the contribution from elastic vector meson production is negligible due to its steep $|t|$ dependence. Measurements of high- $|t|$ diffractive vector meson production $\left(\rho^{0}, \phi\right.$ and $\left.J / \psi\right)$ have been performed by the ZEUS Collaboration [5], $J / \psi$ meson production at high $|t|$ has also been studied by the $\mathrm{H} 1$ Collaboration [阿.

In pQCD models, the $|t|$ distributions are relatively hard compared to those observed for lower values of $|t|$ : a power-law behaviour of the $t$-dependence of the cross section is predicted. A model using BFKL exchange describes well the shape and magnitude of the differential cross section as a function of $|t|$ for $\rho^{0}, \phi$ and $J / \psi$ mesons [5]. The pQCD models differ in the predicted energy dependence of $d \sigma / d t$ : two-gluon exchange yields energy independent cross sections [1], DGLAP evolution predicts practically no energy dependence [价, while BFKL evolution leads to cross sections rising significantly with energy [3]. The rise observed in $J / \psi$ photoproduction at $|t|>5 \mathrm{GeV}^{2}$ [6] suggests the onset of BFKL dynamics at large $|t|$. The violation of $s$-channel helicity conservation (SCHC), the approximation in which the vector meson retains the helicity of the photon, observed in the $\rho^{0}$ data [5], is predicted by the calculations [目].

This proceedings contribution reviews recent HERA measurements of diffractive photoproduction of $\rho^{0}$ mesons at $|t|$ up to $10 \mathrm{GeV}^{2}$ by the $\mathrm{H} 1$ Collaboration [才] and of proton-dissociative photoproduction of $J / \psi$ mesons at $|t|$ up to $20 \mathrm{GeV}^{2}$ by the ZEUS Collaboration [8].

\section{Diffractive photoproduction of $\rho^{0}$ mesons}

The process under study is:

$$
e+p \rightarrow e+\rho^{0}+Y ; \quad \rho^{0} \rightarrow \pi^{+}+\pi^{-},
$$

where the scattered proton is mainly excited into a system $Y$ of mass $M_{Y}$, with only a small contribution from elastic scattering.

The data were collected in the year 2000 with the H1 detector [9] at HERA, which operated with proton and positron beam energies of $920 \mathrm{GeV}$ and $27.5 \mathrm{GeV}$, respectively, and corre- 
spond to an integrated luminosity of $20.1 \mathrm{pb}^{-1}$. The kinematic domain of the measurement is: $Q^{2}<0.01 \mathrm{GeV}^{2}, 75<W<95 \mathrm{GeV}, 1.5<|t|<10 \mathrm{GeV}^{2}, M_{Y}<5 \mathrm{GeV}$.

\subsection{Event selection, reconstruction and Monte Carlo simulation}

The signature of diffractive $\rho^{0}$ photoproduction consists of two oppositely charged pions from the $\rho^{0}$ decay, the dissociated or scattered proton, and the positron scattered in the backward direction. Such events were selected by requiring appropriate energy deposition in the $44 \mathrm{~m}$ tagger, a crystal calorimeter which detects the scattered positron in the backward direction at $44 \mathrm{~m}$ from the interaction point; the reconstruction in the central tracking detector of two oppositely charged particles (pion candidates); the absence in the main calorimeter of any signal with energy larger than $400 \mathrm{MeV}$, not associated with the pion candidates. This cut reduces backgrounds coming from systems decaying into two charged and additional neutral particles. It also limits the mass of the proton dissociative system to $M_{Y}<5 \mathrm{GeV}$.

The $\rho^{0}$ momentum was calculated as the sum of the momenta of the two charged pion candidates. Since the typical $Q^{2}$ is small it was neglected in the reconstruction of the other kinematic variables. In particular, $|t|$ was assumed to be equal to the $\rho^{0}$ transverse momentum squared.

The angle, $\phi^{*}$, between the $\rho^{0}$ production plane and the positron scattering plane was reconstructed in the $\gamma p$ centre-of-mass system, while the polar angle, $\theta^{*}$, of the positively charged decay pion was measured in the $\rho^{0}$ rest frame with the quantisation axis taken as opposite to the scattered proton direction.

A Monte Carlo simulation based on the DIFFVM program [13] was used to correct the data for acceptance and smearing effects. In the simulation, the $t, \cos \theta^{*}$ and $\phi^{*}$ distributions were tuned to those of the data. DIFFVM was also used to simulate the $\omega, \phi$ and $\rho^{\prime}$ backgrounds, which can fake $\rho^{0}$ production through some of their decay channels.

\section{$2.2 \mathrm{t}$ dependence of the cross section and helicity analysis}

The $|t|$ dependence of the normalised differential cross section $1 / \sigma d \sigma\left(\gamma p \rightarrow \rho^{0} Y\right) / d|t|$, presented in Fig. 17a, is well described by the BFKL model of Poludniowski et al. [2], where the model parameters have been taken from the previous ZEUS measurement [5]. A fit to a power law of the form $d \sigma / d t \propto|t|^{-n}$ gave $n=4.41 \pm 0.07$ (stat.) ${ }_{-0.05}^{+0.07}$ (syst.). The difference with the value $n=3.21 \pm 0.04$ (stat.) \pm 0.15 (syst.), measured by the ZEUS Collaboration, is related to the different $M_{Y}$ ranges ( $M_{Y}<25 \mathrm{GeV}$ in the ZEUS case).

The normalised two-dimensional angular distribution $1 / \sigma d^{2} \sigma /\left(d \cos \theta^{*} d \phi^{*}\right)$ can be written in terms of the spin density matrix elements $(\mathrm{SDME}) r_{00}^{04}, r_{1-1}^{04}, \operatorname{Re}\left\{r_{10}^{04}\right\}$. Under the assumption of SCHC, the photoproduced $\rho^{0}$ is expected to be fully transversely polarised and $r_{00}^{04}, r_{1-1}^{04}$ and $\operatorname{Re}\left\{r_{10}^{04}\right\}$ are expected to be zero. The SDME were extracted, for three ranges of $t$, by a fit to the single differential distributions in $\cos \theta^{*}$ and $\phi^{*}$. The extracted values of $r_{00}^{04}$ and $r_{1-1}^{04}$ are presented as a function of $|t|$ in Fig. 1 $\mathrm{b}$ and show a good agreement with the corresponding results from the ZEUS Collaboration. The results for $r_{00}^{04}$ are close to zero, indicating that the production is dominated by transversely polarised $\rho^{0}$ mesons. The results for $r_{1-1}^{04}$ significantly differ from zero, confirming violation of SCHC. The BFKL model of Poludniowski et al. describes $r_{00}^{04}$ well, but the prediction for $r_{1-1}^{04}$ is too large at low values of $|t|$. 


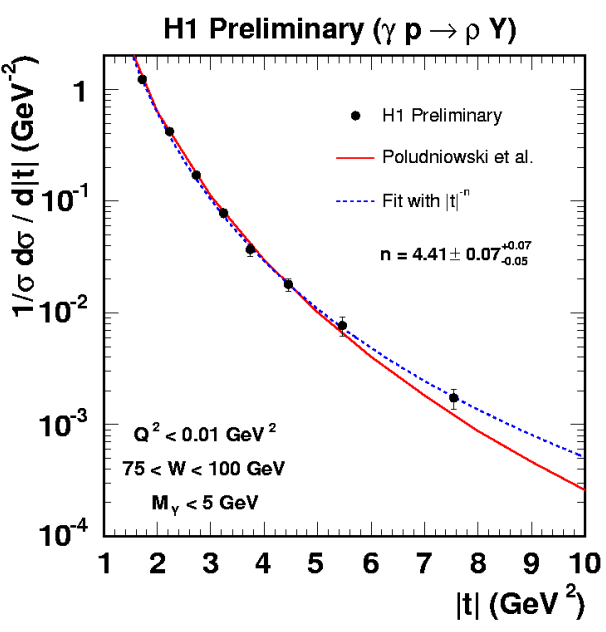

(a)
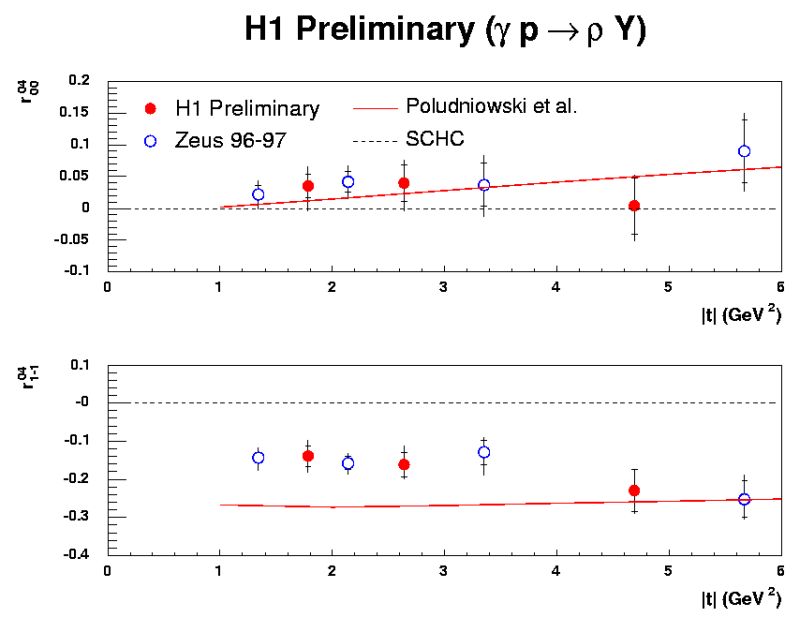

(b)

Figure 1: (a) The $t$ dependence of $\gamma+p \rightarrow \rho^{0}+Y$ normalised differential cross section. The inner error bars show the statistical errors, while the outer ones show the statistical and systematic errors added in quadrature. (b) Measurements of the $r_{00}^{04}$ (top) and $r_{1-1}^{04}$ (bottom) spin density matrix elements as a function of $|t|$ (full points) together with the previous ZEUS measurements [5] (open points). The inner error bars show the statistical errors, while the outer ones show the statistical and systematic errors added in quadrature.

\section{ZEUS}

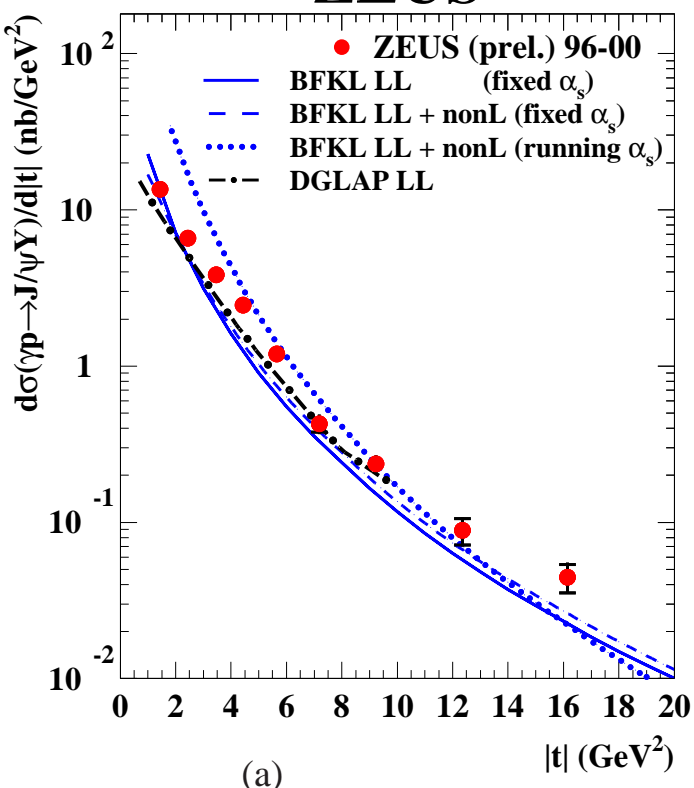

\section{ZEUS}

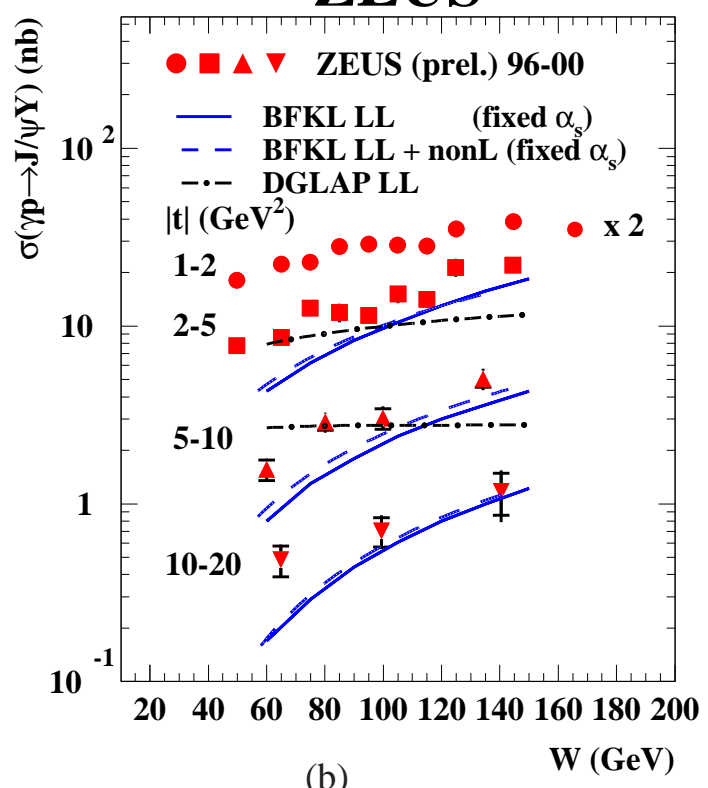

Figure 2: (a) The $t$ dependence of $\gamma+p \rightarrow J / \psi+Y$ differential cross section. The inner error bars show the statistical errors, while the outer ones show the statistical and systematic errors added in quadrature. (b) The $\gamma+p \rightarrow J / \psi+Y$ cross section as a function of $W$ in four intervals of $|t|$. The inner error bars show the statistical errors, while the outer ones show the statistical and systematic errors added in quadrature. 


\section{Diffractive photoproduction of $J / \psi$ mesons}

The process

$$
e+p \rightarrow e+J / \psi+Y ; \quad J / \psi \rightarrow \mu^{+}+\mu^{-},
$$

where $Y$ is the dissociated proton system, has been studied on a sample of data, corresponding to an integrated luminosity of $112 \mathrm{pb}^{-1}$, collected in 1996-2000 with the ZEUS detector [10] at HERA, which over these four years operated with a proton beam energy of $820 / 920 \mathrm{GeV}$ and a positron/electron beam energy of $27.5 \mathrm{GeV}$. The measurement covers the range $50<W<150 \mathrm{GeV}$, $Q^{2} \simeq 0.07 \mathrm{GeV}^{2}, M_{Y}<30 \mathrm{GeV}$ and extends up to $|t|=20 \mathrm{GeV}^{2}$.

\subsection{Event selection, reconstruction and Monte Carlo simulation}

The signature of diffractive $J / \psi$ photoproduction consists of two oppositely charged muons from the $J / \psi$ decay, the dissociated proton, the electron scattered through a small angle and escaping undetected down the beam pipe. The selection procedure required two energy deposits in the main calorimeter (CAL) associated with the two muon candidates from the $J / \psi$ decay; an energy deposit above $400 \mathrm{MeV}$ with $\eta>2.1$ in the forward part of the CAL; a signal in the proton remnant tagger (PRT, for the 1996-1997 data sample) or an energy deposit above $1 \mathrm{GeV}$ in the forward plug calorimeter (FPC, for the 1998-2000 data sample). Energy deposits above the noise level (300 MeV) with $\eta<2.1$, not associated with lepton candidates, were rejected. In order to compare the cross sections with theoretical predictions and previous measurements, an additional cut $z>0.95$ was applied, with $z$ the fraction of the virtual photon energy transferred to the $J / \psi$ in the proton rest frame. This cut limits the mass of the proton dissociative system to $M_{Y}<30 \mathrm{GeV}$.

The kinematic variables were calculated using the momenta of the $J / \psi$ products. Since the typical $Q^{2}$ is small it was neglected in the reconstruction of the other kinematic variables. In particular, $|t|$ was assumed to be equal to the squared transverse momentum of the di-muon system.

The acceptance and the detector effects were determined using the event generator EPSOFT [11]. The simulated FCAL and FPC energy distributions and the $|t|$ distribution were tuned on the data. The generator GRAPE-DILEPTON 1.1 [12] was used to simulate the non-resonant background due to the $\gamma \gamma$ fusion process, $e p \rightarrow e \mu^{+} \mu^{-} Y$, where one of the photons is emitted by the electron and the second photon is radiated off the proton.

\section{2 $\mathrm{t}$ and $\mathrm{W}$ dependence of the cross section}

The differential cross section $d \sigma(\gamma p \rightarrow J / \psi Y) / d|t|$, presented in Fig. 2 2 a, was compared to the predictions from different $\mathrm{PQCD}$ models. The model based on BFKL evolution [B] provides calculations in the leading logarithmic approximation (LL) with and without non-leading corrections (nonL). In the former case predictions for both fixed and running $\alpha_{s}$ are available. The model based on DGLAP evolution [ 4 gives prediction in the LL approximation in the range of model validity $\left(|t|<M_{J / \psi}^{2}\right)$. In all cases the model parameters were taken from the previous ZEUS measurement [5]. The DGLAP, BFKL LL and BFKL nonL predictions with fixed $\alpha_{s}$ are able to describe the shape of the cross section, but not its magnitude, while the BFKL nonL with running $\alpha_{s}$ gives a $|t|$ dependence steeper than that of the data.

The $W$ dependence of the cross section was measured in four bins of $|t|$, as shown in Fig. Z $\mid \mathrm{b}$. The fit to a power law of the form $\sigma \propto W^{\delta}$ resulted in values of $\delta$ increasing with rising $|t|$, 
similar to what previously reported by $\mathrm{H} 1$ [6]. The data of Fig. Zb were also compared to the theoretical predictions (in the case of BFKL-based models, only for fixed $\alpha_{s}$ ). The DGLAP LL calculations are not able to describe the measurements while the BFKL LL predictions qualitatively describe the rise of the cross section. According to Regge formalism $d \sigma / d t \propto W^{4\left(\alpha_{P}(t)-1\right)}$, with $\alpha_{\mathbb{P}}(t)=\alpha_{\mathbb{P}}(0)+\alpha_{\mathbb{P}}^{\prime} \cdot t$ the Pomeron trajectory: a Regge fit to the four $t$ bins yielded an intercept $\alpha_{\mathbb{P}}(0)=1.153 \pm 0.048$ (stat.) \pm 0.039 (syst.) and a slope $\alpha_{\mathbb{P}}^{\prime}=-0.020 \pm 0.014$ (stat.) \pm 0.010 (syst.), consistent with the $\mathrm{H} 1$ measurement [6].

\section{Conclusions}

The recent measurements of diffractive photoproduction of $\rho^{0}$ and $J / \psi$ mesons performed by the ZEUS and the H1 Collaborations at HERA confirm that vector meson production at large values of the momentum transfer $|t|$ shows features of a hard process, reflected in a hard $|t|$ distribution and in the steep rise of the cross section with $W$. In the photoproduction of $\rho^{0}$ mesons, measured up to $|t|=10 \mathrm{GeV}^{2}$, the cross section exhibits a power-like $|t|$ dependence well described by the BFKL model of Poludniowski et al. [2]. The same model reproduces the small measured single spin flip amplitude, but gives only a qualitative description of the large double flip amplitude, providing experimental evidence of $s$-channel helicity non conservation. In the photoproduction of $J / \psi$ mesons, measured up to $|t|=20 \mathrm{GeV}^{2}$, the cross section rises as $W^{\delta}$, with $\delta$ increasing with $t$, suggesting the onset of BFKL dynamics.

\section{References}

[1] V. Barone and E. Predazzi, High-Energy Particle Diffraction, Springer Verlag, Heidelberg, 2002.

[2] R. Enberg et al., JHEP 309 (2003), 008; G. G. Poludniowski et al., JHEP 312 (2003), 002.

[3] R. Enberg et al., Eur. Phys. J. C 26 (2002), 219.

[4] E. Gotsman et al., Phys. Lett. B 532 (2002), 37.

[5] S. Chekanov et al., ZEUS Collaboration, Eur. Phys. J. C 26 (2003), 389.

[6] A. Aktas et al., H1 Collaboration, Phys. Lett. B 568 (2003), 205.

[7] paper n. 640 submitted to this conference.

[8] paper n. 334 submitted to this conference.

[9] I. Abt et al., H1 Collaboration, Nucl. Instr. Meth. A 386 (1997), 310.

[10] ZEUS Collaboration, U. Holm (ed.), The ZEUS Detector. Status Report (unpublished), DESY (1993).

[11] M. Kasprzak, Ph. D. Thesis, Warsaw Univ., DESY F35D-96-16, 1996; L. Adamczyk, Ph. D. Thesis, Cracow Univ. of Mining and Metallurgy, DESY-THESIS-1999-045, 1999.

[12] T. Abe, Comp. Phys. Comm. 136 (2001), 126.

[13] B. List and A. Mastroberardino, Proceedings of the Workshop on Monte Carlo Generators for HERA Physics, A. T. Doyle et al. editors, DESY-PROC-1999-02 (1999), 396. 